\title{
La distribución tradicional del agua del río Turia entre las acequias de la Huerta de Valencia: Un sistema de origen bajomedieval
}

\author{
FERRAN ESQUiLACHE
}

\begin{abstract}
PALABRAS CLAVE: irrigación, distribución del agua, cambios tecnológicos, al-Ándalus.
\end{abstract}

CÓDIGOS JEL: Q15, Q18, Q25, Q55.

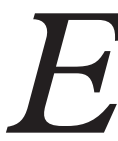

l agua del río Turia se ha repartido tradicionalmente entre las ocho acequias de la Huerta deValencia mediante un sistema de 138 filas, que es una medida de aforo de agua cuya naturaleza no está demasiado clara. También se desconoce cuál es el origen histórico de este sistema de división del agua, que es lo que se intenta resolver en este articulo. Thomas $F$ Glick defendió que se trataba de un sistema de origen andalusi, y propuso una reconstrucción del sistema inicial basado en los turnos horarios, pero aqui se va a intentar demostrar mediante diversos argumentos que esta propuesta no puede ser correcta, especialmente porque se basa en una interpretación errónea de la naturaleza de la fila. Posteriormente se aporta una propuesta alternativa sobre el origen del sistema de repartición en 138 filas que está basada en la proporcionalidad existente entre la cantidad de agua que le corresponde a cada acequia y la cantidad de tierra irrigada por cada sistema hidráulico, y asi se llega a la conclusión de que el sistema de repartición del agua en la Huerta de Valencia solo pudo ser establecido poco después de la conquista cristiana del siglo XIII, cuando la Huerta fue ampliada y reconstruida en gran medida. Finalmente, se concluye que esta es una prueba más de los grandes cambios que se produjeron en los espacios de cultivo irrigado de origen andalusi tras las conquistas cristianas, contrariamente a lo que se venía creyendo hasta ahora. 


\section{The Traditional Distribution of the Water of the Turia River among the Irrigation Canals of the Huerta de Valencia: A Late Medieval System}

\section{KEYWORDS: irrigation, water distribution, technological changes, al-Andalus.}

\section{JEL CODES: Q15, Q18, Q25, Q55.}

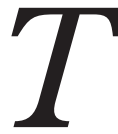

he water of the Turia river has been traditionally distributed in the Huerta of Valencia among eight irrigation canals through a system of 138 filas, a measure of water capacity which nature is not clear. It is also unknown what is the historical origin of this system of water division and this is what we study in this article. Thomas F. Glick defended that it was a system of Andalusi origin, and proposed a reconstruction of the original system based on time shifts, but we try to demonstrate that this proposal cannot be correct, especially because it is based on a wrong interpretation of the nature of the row. Subsequently, we provide an alternative proposal on the origin of the distribution system in 138 filas that is based on the proportionality between the amount of water that each irrigation canal has and the amount of land irrigated by each hydraulic system, concluding that the distribution system of the Huerta deValencia can only have been established shortly after the Christian conquest of the thirteenth century, when the Huerta was greatly expanded and rebuilt. Finally, we conclude that this is another evidence of the great changes that took place in irrigated cultivation spaces of Andalusi origin after the Christian conquests, contrary to what had been said so far.

Recepción: 2020-02-18 - Revisión: 2020-07-08 • Aceptación: 2020-08-12

Ferran Esquilache [orcid.org/0000-0003-2971-2469] es investigador posdoctoral en la Universitat faume I de Castelló. Dirección para correspondencia: Departamento de Història, Geografia i Art, Facultat de Ciències Humanes $i$ Socials, Avenida de Vicent Sos Baynat, s/n, 12071, Castelló de la Plana (Castellón). C.e. ferran.esquilache@gmail.com 


\section{INTRODUCCIÓN}

El agua del río Turia que llega a la Huerta de Valencia y se distribuye entre sus acequias o sistemas hidráulicos se ha contabilizado históricamente mediante una medida de aforo llamada fila. De hecho, existe una distribución tradicional -y en teoría oficial, aunque no esté así especificado en ningún documento conocido- que indica la cantidad de filas de agua del río que corresponden a cada una de las ocho acequias que forman la Huerta, que en total suman 138 filas (Tabla 1).

TABLA 1

Filas de las acequias de la Huerta de Valencia

\begin{tabular}{lr}
\hline Acequia & Filas \\
\hline Montcada & 48 \\
Quart & 14 \\
Tormos & 10 \\
Mislata & 10 \\
Mestalla & 14 \\
Favara & 14 \\
Rascanya & 14 \\
Rovella & 14 \\
Total & 138 \\
\hline
\end{tabular}

Fuente: Cavanilles (1795: 132), Jaubert de Passa (1844-t2: 229); Glick (1988: 304).

El problema es que no conocemos la antigüedad de esta distribución tradicional, y ni siquiera podemos estar totalmente seguros de qué es una fila ni la cantidad de agua que representa. Tradicionalmente se había afirmado que se trata de una medida de aforo del agua en movimiento que pasa por un orificio o por un canal con un tamaño concreto, pero todos los autores que han venido tratando este tema desde mediados del siglo XIX han defendido que, en realidad, se trata de una medida proporcional con respecto al total de un caudal determinado. Entre ellos, el más conocido e influyente ha sido Thomas F. Glick, que, por la novedad que supuso su trabajo en los años setenta del siglo Xx, se convirtió en el autor de referencia para todos los investigadores que se han ocupado en las últimas décadas de la irrigación histórica en España y, en consecuencia, es quien más ha contribuido a difundir esta idea.

También ha sido Glick (1988: 305) quien ha defendido con más argumentos que la distribución tradicional del agua del Turia entre las ocho acequias de Valencia tiene un origen andalusí; $y$, aunque es cierto que sus explicaciones no eran definitivas y ha habido discrepancias, su posición es la más aceptada actualmente. Sin embargo, por ahora no se 
ha llegado a esclarecer de una forma definitiva ni la naturaleza de la fila ni el origen de la distribución tradicional del agua del Turia, y ello está lastrando el conocimiento que tenemos actualmente sobre la forma de distribuir el agua de riego en los diversos períodos históricos. Pero no solo en Valencia, pues, debido a la influencia que aún ejerce el importante trabajo de Glick, hay muchos autores que han trasladado a otros lugares las propuestas de este autor sobre la distribución del agua en los riegos valencianos como si se tratase de informaciones ya demostradas, y no solo de hipótesis de trabajo, de manera que se generan problemas al realizar comparaciones entre sistemas y territorios. En consecuencia, es necesario tratar de esclarecer cuál es el origen de esta distribución del agua para la irrigación, porque la propuesta andalusí de Glick es la única que se ha planteado por ahora y no hay alternativa. Sin embargo, con el avance de la investigación ya hace tiempo que se viene comprobando que tras la conquista cristiana se hicieron cambios en la gestión y organización de la irrigación mucho más profundos de lo que parecía intuirse hasta ahora, y resolver el origen de la distribución tradicional del agua del Turia en la Huerta de Valencia puede aportar bastante información al respecto.

\section{LA FILA DE AGUA COMO MEDIDA DE AFORO: UNA TRIPLE CONDICIÓN}

Para saber cuál es el origen del sistema tradicional de distribución del agua, y el de las conocidas 138 filas de la Tabla 1, primero hay que determinar qué es exactamente una fila de agua. En otro trabajo he intentado explicar con más detalle cuál es el origen y la naturaleza de esta medida de aforo de agua (Esquilache, 2021), por lo que ahora solo intentaré resumir las ideas principales al respecto para poder entender a continuación la explicación sobre el origen de la distribución del agua del Turia.

Como hemos visto, aunque siempre se había pensado que una fila era el agua que pasaba por un orificio con una medida determinada, Maurice Aymard (1864) propuso a mediados del siglo XIX que la fila era una medida de aforo proporcional, una parte alícuota de un caudal determinado. Glick (1988: 298-306) defendió posteriormente esta misma idea mediante la comparación de los sistemas de irrigación valencianos con otros del mundo islámico, aunque se basó especialmente en una sentencia arbitral dictada en 1347 que establecía la distribución del agua del río Mijares, en la que se dice que del caudal del río "sien feytes sexanta files o parts eguals»; y también que estas debían ser repartidas proporcionalmente entre las cuatro villas de la Plana de Castelló según un número determinado de filas para cada una, cuya distribución se puede ver en la Tabla 2. Además, en caso de escasez, toda el agua sería para una sola villa durante dos horas por cada fila asignada en el reparto. Así pues, según lo interpreta Glick, en este documento se dice que 
la fila es $1 / 60$ parte del caudal del río Mijares, y que esta se convertía en dos horas de agua en el supuesto de que hubiese una disminución del agua por sequía.

TABLA 2

Reparto en filas del río Mijares según la sentencia de 1347

\begin{tabular}{lrr}
\hline Localidad & Filas & Horas \\
\hline Vila-real & 14 & 28 \\
Castelló & 14,5 & 29 \\
Almassora & 12,5 & 25 \\
Borriana & 19 & 38 \\
Total & 60 & 120 \\
\hline
\end{tabular}

Fuente: Glick (1988: 302).

El problema es que Glick no parece haber entendido bien este documento, porque cuando se lee con atención la sentencia al completo, se puede ver que no existían dos únicos supuestos para la fila, sino tres: la normalidad, la sequía y la sequía extrema. En el primer supuesto, donde se indica la distribución del agua en condiciones normales de caudal (Tabla 2), la naturaleza de la fila resulta ambigua, porque se da por sabida y no se explicita. Sin embargo, el segundo supuesto prevé, en caso de una disminución del agua disponible, la división del caudal en 60 partes iguales y su reparto entre las cuatro villas según el número de filas que les corresponden, sin ser esta disminución todavía tan grave como para activar el tandeo horario del tercer supuesto previsto para sequía extrema ${ }^{1}$. Por eso se puede afirmar que la fila no puede ser en origen una medida proporcional, es decir, una parte alícuota sobre el total del caudal, puesto que esta condición solo la adquiere durante el segundo supuesto de disminución del agua disponible, de manera que en condiciones normales la fila tiene que ser necesariamente una cantidad concreta de agua. De hecho, esa cantidad de agua no puede ser otra que la capacidad total de la toma de cada acequia junto al azud, pues es físicamente imposible tomar más agua de la que permite el tamaño de la boca de cada acequia sin que el canal se desborde.

1. Después de explicar el primer supuesto ambiguo de normalidad y las filas que corresponden a cada villa, la sentencia dice: "en altra manera, si no bastaven a LX files, que·n sien feytes sexanta parts eguals, e que aquelles sien partides segons que damunt és declarat. E [...] sie feyta partició de la dita aygua per tots temps en cars de necessitat o minva d'aygua». Es decir, que ante una situación de penuria la fila pasaba a convertirse en una parte proporcional de un todo, siendo antes otra cosa.Y, posteriormente, aún añade que "si l'aygua del dit riu venia a tan gran minva o fretura, que segons la partició desús dita la part que pervendrà a la vila d'Almaçora no bastava a una fila, que en aquell cars tota l'aygua del dit riu fos donada a una céquia» por un tiempo concreto, convirtiéndose así cada fila en dos horas de agua en exclusiva. 
En conclusión, la sentencia arbitral del Mijares de 1347 deja bien claro que la fila, al menos en ese momento y lugar, tiene una triple condición que va cambiando de acuerdo con el volumen de agua que hubiese en el río. Siendo así, según el caso, (i) una cantidad de agua concreta - por ahora desconocida- pasando por el cauce del río en un punto, (ii) una cantidad proporcional del agua total que hay en el río en ese punto, o bien (iii) una equivalencia horaria que vale por dos horas de agua cada fila. Por tanto, es muy factible que esta triple condición que se documenta en el siglo XIV se pueda retrotraer a época andalusí, y probablemente a todos los períodos y lugares. De hecho, existen diversos documentos de época medieval en los que se habla de filas de agua que, por si solos, no permiten llegar a esta conclusión por su ambigüedad, pero que cuando se leen desde esta triple perspectiva son bastante claros (Esquilache, 2021).

Por lo que respecta al tamaño del orificio, o del canal, que en condiciones normales sirve para medir cuanta agua es una fila, no existen demasiadas referencias, pero con las que hay es factible proponer que, al menos para época moderna, en Valencia una fila era el agua que pasaba por un orificio cuadrado con un palmo foral de longitud en cada lado $(0,2265 \mathrm{~m})$. Así al menos lo consideraba, por ejemplo, entre otros, el ingeniero hidráulico Jerònim Negret cuando realizó en 1617 un conocido informe perital sobre el tamaño de las bocas de todas las acequias del Turia desde su cabecera hasta la Huerta de Valencia, que es conocido en la historiografía como el expediente Sisternes por el nombre del fiscal de la Audiencia de Valencia que se lo encargó, Melchor Cisternes d'Oblites. Sin embargo, hay otros documentos que apuntan a la existencia de medidas distintas para la fila. Por ejemplo, una especie de «manual» escrito en 1631 por uno de los encargados de medir el nivel del río Mijares y hacer cumplir la referida sentencia de 1347, donde se dice que en Castellón la fila, que además llaman fila real, es el agua que pasa por un canal con tres palmos de ancho y uno de alto, es decir, el agua que pasa por tres palmos cuadrados $\left(0,1539 \mathrm{~m}^{2}\right)$; el triple que la fila habitual de un solo palmo cuadrado $\left(0,0513 \mathrm{~m}^{2}\right)$. Por lo tanto, parece que habría más de una medida para la fila.

Ahora bien, si la fila era la cantidad de agua que pasa por un orificio con un marco de uno o tres palmos cuadrados de los establecidos en 1238 por Jaime I en los Fueros de Valencia, esta no puede ser la medida original, puesto que la fila-cuyo nombre es una traducción del término árabe jayt («hilo»)- es una medida de aforo de origen claramente islámico. Una manera de saberlo podría ser medir alguna infraestructura hidráulica de probable origen andalusí por la que se sepa cuántas filas pasaban, y un ejemplo adecuado pueden ser, precisamente, las bocas de entrada de las acequias de la Huerta de Valencia. Conocemos con seguridad la medida de dos de ellas, Rascanya y Montcada, porque nos la indica François Jaubert de Passa (1844: 250, 418), y, si dividimos la luz de estas bocas entre el número de filas que le corresponden, podemos saber que el marco de la fila usada 
en Rascanya era de un codo andalusí de 30 dedos $(0,52 \mathrm{~m})$ por cada lado del cuadrado ${ }^{2}$. En el caso de Montcada, por el contrario, no está claro qué medida se utilizó para establecer las 48 filas que le corresponden según la distribución tradicional, pero parece plausible que se trata de una fila exclusiva para este canal, y, si se aplica la misma medida que en Rascanya, parece probable que le correspondiesen 24 de las filas de este otro tamaño ${ }^{3}$. En conclusión, el uso de una medida andalusí para calcular el marco de la fila en la acequia de Rascanya -y muy probablemente en el resto de las acequias de la Huerta de Valencia- evidencia que la fila como medida de aforo tenía este origen, pero que en algún momento indeterminado, tras la conquista cristiana, se cambió la forma de medirla, pues en época moderna no se usaba el codo andalusí de 30 dedos $(0,5224 \mathrm{~m})$ en cada lado para medir su marco, sino el palmo foral valenciano $(0,2265 \mathrm{~m})$, que fue establecido por primera vez en los Fueros a finales de 1238.

\section{LA DISTRIBUCIÓN DEL AGUA DEL RÍO TURIA SEGÚN EL MODELO PROPUESTO POR GLICK}

Una vez tenemos claro qué es una fila en sus tres formas cambiantes, y sabemos de manera razonable cuánta agua podría ser una fila en época andalusí y en época foral, es el momento de analizar cómo se aplicaba esta medida de aforo en la distribución del agua del río Turia. Thomas Glick (1988:303-305) planteó que el sistema tradicional de reparto de las 138 filas que veíamos al principio era muy antiguo, aunque este no sería el sistema original, sino una trasformación de un sistema anterior, probablemente de época andalusí. De manera que presentó una nueva propuesta de reconstrucción del supuesto sistema inicial, inspirado en el sistema del segundo y tercer supuestos medioambientales de la sentencia del Mijares, que se basa en la proporcionalidad y en los turnos horarios. De-

2. El cálculo realizado es el siguiente: la boca de entrada de Rascanya, actualmente destruida, tenía 3,3975 m de ancho, y el agua entraba con 1,1325 $\mathrm{m}$ de altura desde el fondo, por lo que la toma tenía una luz de $3,85 \mathrm{~m}^{2}$. Si dividimos esta cifra entre 14 , que son las filas correspondientes a Rascanya, podemos saber que cada fila tenía una luz de $0,275 \mathrm{~m}^{2}$, y con su raíz cuadrada sabemos que esta superficie corresponde a un marco de 0,52 m de lado, que es claramente un codo andalusí de 30 dedos (ESQUILACHE, 2021).

3. El cálculo es el mismo que en Rascanya. Con 5,6625 $\mathrm{m}$ de ancho por 1,2458 $\mathrm{m}$ de altura hacen una luz de 7,054 $\mathrm{m}^{2}$ para la toma de Montcada, que dividida entre 48, que es su número de filas, tenemos que cada fila era de $0,14696 \mathrm{~m}^{2}$, y con su raíz cuadrada salen $0,38 \mathrm{~m}$ de lado. Sin embargo, el marco de esta fila no cuadra con ninguna medida andalusí o foral conocida. Todo indica que esta fila es exclusiva de Montcada, puede que una especie de fila real como las que existen en otros lugares, porque tiene casi la mitad de superficie que la de Rascanya, de lo que se deduce que se trata de una modificación como consecuencia del carácter excepcional de la Real Acequia de Montcada, y que en origen le corresponderían 24 filas de las del mismo tamaño que Rascanya. Véase todo el razonamiento detallado en ESQUILACHE (2021). 
jaba así de lado, sin ni siquiera nombrarlo, el primer y más importante supuesto ambiental de la sentencia de 1346 en el que la fila era la cantidad de agua que pasaba por un orificio con un marco determinado.

Partiendo de la división tradicional del caudal del río en 138 filas distribuidas para las ocho acequias de la Huerta (Tabla 1), este autor propuso que, en origen, existiría una distribución proporcional en la que cada fila correspondía a una parte del caudal total (el segundo supuesto del Mijares) y que la fila se convertía en una hora de agua en exclusiva para cada acequia durante las sequías (como el tercer supuesto del Mijares). Además, como utiliza un modelo horario, dedujo que en el sistema original andalusí no serian 138 filas/horas, sino 144, para completar un ciclo de 6 días. En consecuencia, para Glick, las 48 filas de la Real Acequia de Montcada habrían sido 48/144 partes del caudal del río a la altura de su azud, que en tiempos de escasez se convertirían en 48 horas de agua para esta acequia; es decir, que tendría todo el caudal del río en exclusiva durante dos días seguidos. El resto de las acequias de la Huerta, con 10 o 14 filas cada una según el caso, se repartirían el agua de igual manera pero por parejas (por orden, una de cada lado del río), para sumar entre ellas turnos de 24 horas. Es decir, que en condiciones normales las acequias tendrían 10/144 o 14/144 partes respectivamente del agua del río, y en caso de sequía cada pareja de acequias tendría toda el agua del río durante 10 y 14 horas para cada una, que sumadas son 24 horas, y al día siguiente sería para otra pareja de acequias hasta completar el ciclo de 6 días (Tabla 3).

TABLA 3

Reparto original de las filas de la Huerta según T. F. Glick

\begin{tabular}{lclrc}
\hline Margen derecho & Filas & Margen izquierdo & Filas & Total pareja partes/horas \\
\hline- & - & Montcada & 48 & 48 \\
Quart & 14 & Tormos & 10 & 24 \\
Mislata & 10 & Mestalla & 14 & 24 \\
Favara & 14 & Acequia desaparecida & 10 & 24 \\
Rovella & 10 & Rascanya & 14 & 24 \\
\hline & & & & Total 144 \\
\hline
\end{tabular}

Fuente: Glick (1988: 304).

No obstante, para hacer cuadrar las parejas de acequias y formar turnos conjuntos de 24 horas, y para que todas juntas sumasen 144 filas u horas (que serían 6 días, más uno de descanso que completaría la semana), Glick necesitaba rebajar la dotación de la acequia de Rovella desde sus 14 filas establecidas a solo 10, basándose en un supuesto error tipográfico que podría haber sido cometido en algún momento indeterminado y después arrastrado a lo largo del tiempo por el simple hecho de que todos los autores se copian 
unos a otros. Además, necesitaba añadir una nueva acequia con 10 filas de agua que actualmente estaría "desaparecida", si bien es cierto que con ello no se refería a la desaparición física de todo un sistema hidráulico, sino solo a una comuna de regantes que se hubiese extinguido, y su acequia se hubiese integrado en otra comuna como un brazo secundario, desapareciendo con ella su dotación de agua (Glick, 1988: 305) ${ }^{4}$.

Se trata, en definitiva, de una propuesta inteligentemente planteada que intentaba solucionar un problema histórico -el del origen de la distribución del agua del río Turia en la Huerta de Valencia- para el que no se intuía ninguna solución con los datos disponibles en los años sesenta del siglo Xx, cuando Glick ideó su hipótesis. Con todo, es evidente que se pueden hacer diversas objeciones a la propuesta, y de diferente tipo. Algunas muy concretas, como ya hizo el geógrafo Eugenio Burriel (1971: 163) ${ }^{5}$, pero también otras más importantes, como que se centrase solo en la proporcionalidad.

Por lo que respecta a las objeciones concretas, es evidente que la rebaja de 14 a 10 filas para Rovella no tiene justificación documental alguna, porque en todas las fuentes conocidas donde se recoge el reparto de las 138 filas aparece citada esta acequia con sus 14 filas. La hipótesis de la supuesta acequia "desaparecida» también fue muy criticada, pero en este caso es necesario reconocer que quizás sí hubo una acequia que más tarde se fusionó con otras, y de hecho más de una. Hace algunos años Enric Guinot (2005: 271300), basándose en ciertos indicios morfológicos y pistas documentales, ya propuso que la acequia necesaria para completar la propuesta de Glick podría ser cualquiera de los tres brazos principales en los que se divide la acequia de Mestalla, pues parece que este sistema hidráulico es el resultado de la fusión de tres sistemas distintos, cada uno con su propio azud dentro del cauce del río ${ }^{6}$. Sin embargo, aunque esta fusión de tres sistemas hidráulicos en uno parezca reforzar la tesis horaria de Glick, es evidente que la cuestión de

4. GLICK (1988) añadió en este punto una nota a la traducción española de Irrigation and Society que no estaba en el original inglés, donde argumentaba que este brazo "desaparecido» podría ser el de Rambla, de la acequia de Mestalla, basándose en el hecho de que esta zona pagaba el diezmo por separado en época foral, según le había informado Manuel Ardit. También lo explica en Glick (1989).

5. También lo criticaron Antonio López Gómez (1975) y Eduardo Manzano (1986), pero ambos se basan en la crítica originaria de Burriel.

6. Además de los argumentos documentales y morfológicos aportados por Guinot, que ya eran bastante concluyentes, un detallado análisis morfológico del sistema de Mestalla permite confirmar que en origen se trataba de tres sistemas distintos, e incluso se puede proponer un trazado original en base a la topografía del terreno. El mismo análisis permite proponer también que este cambio se debió producir, posiblemente, para tomar el agua del río antes que la acequia de Rascanya, que es la última que se construyó en la Huerta de Valencia, lo que permite confirmar que la fusión en un solo sistema se produjo ya en época andalusí, posiblemente hacia los siglos XI o XII. Véase una argumentación detallada en ESQUILACHE (2018: 292-299). 
la acequia supuestamente desaparecida no es el único argumento de peso en contra, como ya señaló Burriel (1971: 163).

Es totalmente ilógico que en un momento de sequía, que es cuando se activaba el tandeo horario, se dejase perder el agua de todo un día para el descanso semanal.Y, si bien es cierto que Glick (1989) respondió más tarde que un turno de seis días no es tan extraño - pues en Murcia, según dice, no habría riego los domingos, y las leyes semíticas en general suelen prohibir el riego en el día de descanso-, la respuesta continúa sin ser nada convincente, porque estamos hablando precisamente de medidas contra la escasez. De hecho, en la sentencia del Mijares que hemos visto el tandeo horario que se activa en caso de sequía extrema dura solo cinco días, de manera que cuando acaba el turno vuelve a empezar sin interrupción y sin desperdiciarse ningún día de agua, por lo que el ciclo de una semana completa es totalmente innecesario.

Por otro lado, hasta donde sabemos, la acequia de Montcada nunca ha participado en el tandeo del agua del río. No así las otras siete acequias de la Huerta de Valencia, que, al menos desde época bajomedieval, se turnaban el agua cuando había sequía, pero no por parejas, sino por vertientes. Desde el momento en el que se activaba el tandeo, toda el agua era para las acequias de uno de los lados del río durante dos días, y durante los dos días siguientes era para las acequias del otro lado, y así sucesivamente mientras durase la sequía (Burriel, 1971: 174) ${ }^{7}$. De nuevo, evidentemente, sin días de descanso. Además, esto era así porque el Turia es suficientemente caudaloso como para, incluso con sequía, poder abastecer a varias acequias a la vez. De manera que si en época foral y contemporánea, que es cuando está documentado este tandeo, había suficiente agua para hacerlo así, más debió serlo en época andalusí cuando la superficie irrigada en la Huerta era muchísimo menor (Esquilache, 2018), sin ser necesarios turnos por parejas.

Por lo que respecta a la proporcionalidad como explicación principal del sistema de reparto del agua propuesto por Glick, y su equivalencia con un turno horario ocasional, también se pueden realizar algunas objeciones. En primer lugar, Burriel (1971: 166) ya criticó a Glick porque, según dice, mediante el sistema fluvial de azudes, compuertas y almenaras del Turia no es posible tomar una proporción concreta del caudal del río, y que

7. Esto no significa, por otro lado, que la acequia de Montcada no cediese agua cuando había sequía para las otras de más abajo, pero lo hacía de acuerdo con diversas disposiciones bien conocidas, dictadas por Jaime II en 1321, que posteriormente fueron la base legal de la distribución del agua del río Turia en tiempos de sequía. Parece probable que el rey innovase en ese momento y no recogiese ninguna costumbre anterior, puesto que en 1310, durante otra sequia de menor entidad, el Consell municipal de la ciudad ya había dictado disposiciones parecidas pero distintas (Archivo Municipal de Valencia, Manual de Consells, A1, f. 93r-95r). 
esto solo podría hacerse si existiesen dentro del cauce partidores proporcionales de lengua, es decir, con tajamar, como los que hay dentro de las acequias para dividirlas en diversos brazos. Lo cierto es que, a pesar de lo que dice Burriel, sí es posible tomar el agua proporcionalmente con este sistema de azudes y compuertas, porque, de hecho, en el río Mijares se hacía en cumplimiento de la sentencia de 1346, como además demuestra el manual de partición de 1631. Pero para poder hacerlo se necesitaba gente presente midiendo de forma constante el nivel del río y manipulando las compuertas para poder compensar los cambios en la cantidad de agua disponible, nunca de forma automática como ocurre en los partidores proporcionales que cuentan con un tajamar de piedra y no necesitan atención humana. Por lo tanto, aunque a primera vista este argumento parezca reforzar la propuesta de Glick, en realidad demuestra que el reparto proporcional del río solo puede producirse en momentos excepcionales, cuando hay una sequía que dura por un periodo de tiempo limitado y ni siquiera se produce todos los años, que es el segundo supuesto medioambiental de la sentencia del Mijares de 1346. Pero no se puede producir de forma continua en situaciones normales de caudal, como pretendía Glick, porque esto requeriría una atención humana constante que nunca se produjo.

Por otro lado, la propuesta de Glick sobre la división del caudal del río en 144 partes iguales, convertibles en una división horaria de 24 horas, se basó en una supuesta similitud del sistema valenciano con la distribución del agua del río Barada para la Ghūta de Damasco. Allí, efectivamente, el caudal del río se divide en cada punto en 24 partes iguales, y existe una medida proporcional que es convertible en tiempo, llamada qirāt, que supuestamente equivaldría a la fila valenciana. Por eso, entre otras cosas, Glick (1988: 333) llamó «tipo sirio» al sistema de distribución del agua en la Huerta de Valencia, e incluso llegó a proponer más tarde que podría haberlo ideado e instaurado algún funcionario estatal con este origen (Glick, 1989), para adecuar así su hipótesis a los trabajos de Pierre Guichard sobre la población mayoritariamente bereber de Sharq al-Ándalus. Pero, en realidad, el modelo sirio de Ghūta y el valenciano de la Huerta no son iguales, y solo se parecen con la propuesta de reconstrucción del sistema original de distribución que realizó el propio Glick, porque con el sistema de distribución real que conocemos por la documentación no se parecen en nada, excepto en el hecho de que hay un río y unas acequias que toman el agua mediante azudes.

El qirāt árabe siempre es 1/24 parte de un todo, no solo para el agua, y de él deriva la palabra quilate y el sistema actual para establecer la pureza del oro, por ejemplo. En el caso de Damasco, el qirāt es 1/24 parte del total del caudal del río Barada que hay en cualquier punto de su cauce, o 1/24 parte del agua que hay en un canal cuando llega a un partidor. Es decir, siempre es proporcional, y además convertible en un turno horario de 24 horas, un día. El problema es que Glick extrajo la información sobre la organización de 
la distribución del agua de riego en Ghūta a partir de dos artículos publicados a principios del siglo Xx, cuando Siria era aún una colonia francesa, cuyos autores habían recogido la información mediante encuestas de campo realizadas entre los responsables de controlar la irrigación (Tresse, 1929; Thoumin, 1934). Es decir, se trata del sistema de distribución del agua del río Barada vigente a principios del siglo $\mathrm{xx}$, pero hay bastantes indicios para pensar que este era el resultado de una modificación posterior a la conquista otomana de Siria, en el siglo XVI, y no el sistema original de Ghūta que, presuntamente, se podría haber copiado en Valencia a partir del siglo VIII.

En 1797 el matemático sirio Muhammad al-'Attār escribió una obra titulada 'Ilm al-

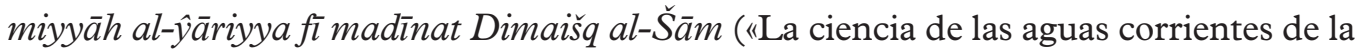
ciudad de Damasco»), en la que explicaba que la distribución de las aguas en la ciudad -que ya era tradicional en aquel momento- estaba basada en el codo turco de Estambul, que se divide en qirāt. Además, aunque en época de al-'Attār, a finales del xviII, la presa de distribución donde se repartía el agua del río Barada entre todos los sistemas ya era vieja, aún pudo encontrar en los archivos de la ciudad un documento que hablaba de la construcción de la presa en el que se explicaba cómo se debía repartir el agua del río de forma proporcional (El Faïz, 2005: 296-299). Difícilmente, pues, el documento podía ser muy antiguo, o al menos no podía serlo tanto como para ser anterior a la llegada de los sirios a al-Ándalus, cuando supuestamente se habría copiado el sistema en Valencia.

En definitiva, todo parece indicar que la supuesta relación entre la fila y la distribución del agua en Valencia con el qirāt y la distribución del agua en Damasco (el modelo sirio de Glick) no tiene tanto fundamento como podía parecer, puesto que el sistema de Ghūta es más tardío, de época otomana. Por el contrario, si observamos más detenidamente el reparto del agua del río Barada que decretó el califa Hishām en el siglo vIII, y que publicó traducido el propio Glick (1988: 384), veremos que no existe referencia alguna a una parte alícuota. De hecho, este documento se interpreta mucho mejor si, en lugar de traducir la palabra maskaba por "parte", como hizo Glick sin explicar muy bien por qué, asumimos que se trata de volúmenes de agua fluyendo por un lugar con un tamaño concreto, similar a los que hemos visto en Valencia para la fila; puesto que la raíz árabe $s-k-b$ hace referencia, como él mismo indica, a la acción de "verter» $\mathrm{o}$ "derramar», en el mismo sentido que jayt («hilo»), y no a una parte alícuota como Glick pretendía. Es, pues, en este otro sentido -en el que la proporcionalidad no parece tener importancia excepto en momentos de excepción- que el modelo de Ghūta en vigor en el siglo viII se parece al modelo de la Huerta de Valencia que nos ha llegado a la contemporaneidad, cuya versión original seguramente también se implantaría en aquellos mismos años. Con todo, es necesario advertir que este modelo no existía solo en Siria, pues estaba más generali- 
zado de lo que parece, porque es también el sistema de distribución del agua en los wadi del Magreb ${ }^{8}$.

Así pues, la propuesta de Glick para Valencia, aunque ingeniosa y válida como hipótesis en su momento, a día de hoy presenta muchos más problemas que soluciones aporta. Además, está muy forzada en diversos aspectos para destacar la proporcionalidad como forma principal de realizar la distribución, y ahora sabemos que esto no fue así porque la proporcionalidad solo se aplicaba en situaciones de excepcionalidad hídrica. En cualquier caso, la duda sigue siendo cuál es el origen de las 138 filas tradicionales y de todo el sistema de distribución del agua del Turia en la Huerta de Valencia, documentado al menos desde época moderna y hasta bien entrado el siglo xx, cuando dejó de ser necesario como consecuencia de la regulación artificial del río mediante la construcción de pantanos de acumulación.

\section{EL ORIGEN DE LAS 138 FILAS DE LA HUERTA DE VALENCIA: UNA PROPUESTA ALTERNATIVA}

Thomas Glick estaba convencido del origen andalusí de las 138 filas con las que se ha distribuido tradicionalmente el agua del Turia entre las ocho acequias de la Huerta (Tabla 1). Por su parte, Eugenio Burriel (1971: 164-167) argumentó que las 138 filas no se pueden retrotraer documentalmente más allá del siglo XVII, por eso defendía que las debió establecer el ingeniero hidráulico Jerónim Negret en su informe para la Audiencia deValencia, conocido como expediente Sisternes por la historiografía (Esquilache, 2021). Así, para Burriel, Negret habría medido las bocas de entrada de las acequias de la Huerta de Valencia asumiendo que la fila era el agua que pasa por un palmo cuadrado foral, y así es como habría establecido por primera vez las 138 filas, antes desconocidas, cuando en realidad la fila debía ser proporcional, como en época andalusí. Pero sabemos que esto no fue así. Es cierto que, por ahora, la referencia más antigua que se conoce a las 138 filas es la del expediente Sisternes, pero no todos los autores que las citan conducen siempre a este documento como su fuente. De hecho, Cavanilles (1795: 132) cita las 138 filas a pesar de no haber conocido el informe de Negret, porque fue Francisco X. Borrull (1831) quien lo localizó en el archivo de la Audiencia de Valencia unos años después de la publicación

8. El mismo GLICK (2007) supo más tarde que este modelo de distribución del agua de riego se encuentra implantado especialmente en el norte de África, el lugar de origen de las tribus bereberes que colonizaron Sharq al-Ándalus tras la conquista islámica de la península Ibérica en 711 . No era necesario, pues, ningún funcionario sirio para implantar el modelo. En realidad, este sistema es el más habitual entre los sistemas hidráulicos fluviales y, sin duda, el que la población bereber ya conocía cuando llegó a al-Ándalus. 
de las Observaciones. Además, ahora sabemos que las filas de la Huerta de Valencia tienen una medida basada en el codo andalusí de 30 dedos, y no de un palmo foral como usaba Negret, de manera que las 138 filas tradicionales de la Huerta son más antiguas.

Ahora bien, que la medida de aforo usada para la distribución de las aguas del río Turia esté basada en una medida de longitud andalusí no quiere decir que necesariamente sea este también el origen de las 138 filas, como pretendía Glick, pues la medida de la fila andalusí se podría haber seguido usando con posterioridad a la conquista cristiana; al menos por un tiempo, aunque, por ahora, no lo podemos saber seguro. No obstante, hay una manera de encontrar ese origen desconocido, $\mathrm{o}$ al menos de realizar una propuesta más afinada y alternativa a la de Glick que, de nuevo, nos la proporcionará una comparación con la sentencia de 1347 para el reparto del agua del río Mijares entre las cuatro villas de la Plana de Castelló.

Parece evidente que las 60 filas con las que contaba el Mijares se establecieron midiendo el caudal habitual del río en un punto determinado, como una especie de nivel medio ante los cambios experimentados a lo largo del año; o puede que sea más bien un mínimo estival en años normales sin sequía. Lo que no especifica la sentencia es cómo se asignó el número de filas que correspondía a cada villa de la Plana, según hemos visto en la Tabla 2. Sin embargo, hace ya algunos años Sergi Selma (2005: 403) pudo establecer cómo se hizo esta distribución gracias al citado «manual» de repartición de 1631, puesto que este recoge la cantidad de tierra irrigada que fue estimada para cada una de las villas de la Plana en el momento de hacer la repartición pactada en la sentencia de 1347. De hecho, las cifras de superficie se dan en jovades (una jovada $=2,99 \mathrm{ha}$ ), que era una medida de superficie agraria foral de uso habitual en época medieval, pero poco usual en época moderna por la reducción de las explotaciones. Así pues, como indica Selma (2005), si se divide el número de jovades que se regaban en cada una de las villas de la Plana en 1347 -según recoge el documento de 1631- entre el número de filas de agua que les asignaron a cada villa en la sentencia, el resultado es exactamente de 50 jovades de tierra por cada fila de agua.

En consecuencia, es evidente que el número de filas asignadas en la Plana estaba en relación con la superficie de tierra irrigada por cada sistema hidráulico, independientemente de su antigüedad anterior o posterior a la conquista ${ }^{9}$, de la situación de su azud en el río aguas arriba o abajo, o de la vertiente fluvial en la que estuviese el sistema. De hecho, la base legal de esta repartición es muy clara, y está en el fuero 376 (rúbrica XLVIII)

9. La acequia de Vila-real la mandó construir Jaime I ex novo al mismo tiempo que fundaba la villa, pero las acequias de Borriana y de Castelló-Almassora ya existían en época andalusí. 
de los Fueros de Valencia establecidos por Jaime I en 1238, el cual dice que «l'aygua de flum públich deu ésser partida segons la manera e la granea de les possessions a-rregar los camps» ${ }^{10}$. Así pues, puesto que era lo que marcaba la ley y sabemos que, efectivamente, se hizo así, al menos, en el caso del Mijares, parece probable que también se hiciese así en el resto de los ríos valencianos con diversos sistemas de irrigación, aunque no tengamos la prueba documental concreta, como ocurre en la Plana de Castelló.

En el caso de la Huerta de Valencia conocemos el número de filas asignadas a cada sistema hidráulico según la distribución tradicional de 138 filas (Tabla 1), pero no sabemos cuánta tierra irrigaba cada uno de ellos inicialmente, hasta que Jaubert de Passa nos aporta datos de superficie irrigada en el siglo XIX extraídos de registros fiscales en los archivos de las comunas que pudo consultar ${ }^{11}$. Es bien sabido que en época moderna los sistemas hidráulicos de la Huerta se ampliaron en mayor o menor medida -aunque no se ha estudiado exactamente cuánto-, de manera que los datos de superficie aportados por Jaubert de Passa no pueden ser los originales de cada sistema, pero, como no disponemos de otros más antiguos, los del siglo XIX pueden servir para hacer una primera aproximación. El resultado es la Tabla 4, en la que se compara por columnas el porcentaje de agua del río que recibía cada acequia según el reparto clásico de filas, con el porcentaje de tierra regada por cada acequia con relación a la suma total de tierras irrigadas por todas ellas. Y, finalmente, la última columna es el resultado de dividir la superficie regada en el siglo XIX (expresada en jovades forales) entre el número de filas que le corresponden según el reparto tradicional. Ahora bien, puesto que las 48 filas de Montcada no se miden del mismo modo que las del resto de las acequias (véase nota 3), se le han asignado a esta acequia solo 24 filas, que son las que probablemente le corresponden en filas medidas con el marco de un codo andalusí de 30 dedos, de manera que la suma total de filas en la Tabla 4 no son 138, como tradicionalmente han sido, sino 114 filas $^{12}$.

10. Esto, de hecho, es derecho romano, basado en el Digesto de Justiniano del año 530, el cual dice justo lo mismo pero en latín. Véase la introducción de Arcadi García al libro de V. García Edo (1994: 15).

11. El cuadro sinóptico donde se recogen los datos en conjunto lo elaboró Francisco Alguer en 1828 a partir de la obra original francesa de Jaubert de Passa, y fue incluido en el volumen 2 de la traducción castellana (Jaubert de Passa, 1844: t. 2, 229). Probablemente se trata de datos pertenecientes a momentos diferentes, pero, si los comparamos con los que aporta Maurice Aymard (1864) unos años después, son casi idénticos, con la única excepción de Montcada, puesto que Jaubert de Passa debió tomar la cifra de algún documento antiguo del archivo, mientras que Aymard seguramente aporta las cifras reales del momento de confección de su obra.

12. Es importante advertir, no obstante, que si hacemos los cálculos manteniendo las 48 filas de Montcada, sumando en total 138, obviamente los resultados son algo distintos, pero la interpretación que se puede hacer de ellos no difiere en demasía, por lo que el cambio no afecta a las conclusiones de este trabajo. 
TABLA 4

Filas de la Huerta en relación con la superficie irrigada en el siglo XIX (en jovades)

\begin{tabular}{lrrrrc}
\hline Acequia & Filas & \%filas & Superficies. XIX & \% superficie & Jovades por fila \\
\hline Montcada & 24 & $21,1 \%$ & 1.066 & $30,4 \%$ & 47,56 \\
Quart & 14 & $12,3 \%$ & 515 & $14,7 \%$ & 36,79 \\
Tormos & 10 & $8,7 \%$ & 305 & $8,7 \%$ & 30,50 \\
Mislata & 10 & $8,7 \%$ & 283 & $8,1 \%$ & 28,30 \\
Mestalla & 14 & $12,3 \%$ & 387 & $11,0 \%$ & 27,64 \\
Favara & 14 & $12,3 \%$ & 519 & $14,8 \%$ & 37,07 \\
Rascanya & 14 & $12,3 \%$ & 262 & $7,5 \%$ & 18,71 \\
Rovella & 14 & $12,3 \%$ & 172 & $4,9 \%$ & 12,28 \\
Total & 114 & $100,0 \%$ & 3.509 & $100,0 \%$ & 30,25 \\
\hline
\end{tabular}

Fuente: Elaboración propia con datos tomados de Jaubert de Passa (1844-t2: 229).

Lo primero que llama la atención es que existe una cierta proporcionalidad entre el porcentaje de filas de cada acequia en relación con el total del río, con el porcentaje de tierra regada por cada acequia en relación con total de la Huerta. Es decir, a más filas de agua disponibles por cada acequia, más superficie irrigada, y a la inversa. De hecho, en el caso de Tormos el porcentaje de agua y tierra es el mismo, mientras que en el caso de Mislata y Mestalla es muy cercano, y en el resto no difieren de un modo extraordinario. Por otro lado, es muy interesante la última columna, en la que se refleja el número de jovades de superficie que se regaban en el siglo XIX por cada fila de agua disponible.Y, aunque la cantidad de tierra por fila difiere en todos los casos -lo que es muy normal, porque se trata de cifras de superficie muy tardías y el aumento de la superficie irrigada fue distinto en cada caso-, sí se puede apreciar que la mayoría están entre las 27-28 jovades por fila de Mestalla-Mislata y las 36-37 jovades por fila de Quart-Favara, con la excepción de Moncada, que son 47 jovades, y de Rascanya y Rovella, que están muy por debajo con 18 y 12 respectivamente. Mientras que Tormos, con 30 jovades por fila, está justo en la media del conjunto de la Huerta.

Con estos datos parece deducirse que, efectivamente, algunos sistemas hidráulicos habían aumentado la superficie irrigada desde que se produjo la asignación original de las filas, fuese cual fuese ese momento. Por ejemplo, de forma excepcional tenemos datos medievales de superficie irrigada para la acequia de Tormos, gracias a un pleito en el que fue recogido este dato (Glick, 1988: 59; Guinot \& Selma, 2005: 102), y así sabemos que en 1433 (200 años después de la conquista y 400 antes de los datos de Jaubert de Passa) se regaban 283 jovades con el agua de esta acequia, que son 22 menos de las 305 que aparecen recogidas en el siglo XIX, por lo que esta acequia había crecido muy poco en época moderna. Por otro lado, gracias a un registro fiscal de tierras irrigadas por Favara, ela- 
borado en 1741, sabemos que esta otra acequia regaba 508 jovades en el siglo XVIII, que son solo 11 menos de las que regaría unos cien años después según los datos de Jaubert de Passa ${ }^{13}$. Pero si eliminamos del cómputo la tierra regada en los alters por parades grosses, que fueron añadidos claramente en época moderna forzando el sistema, la cifra total de tierra irrigada por este sistema hidráulico para época bajomedieval se puede calcular en unas 429 jovades (Esquilache, 2014), que son 90 menos de las que ya regaba en el siglo XIX. En el caso de esta última acequia, pues, se había producido un aumento importante de la superficie irrigada.

Esto nos demuestra, en definitiva, que con posterioridad a la división del río en 138 filas los sistemas se ampliaron, aunque relativamente poco en algunos casos. De hecho, se trata de pequeñas ampliaciones dentro del perímetro del sistema, realizadas sobre tierras que estaban situadas por encima del nivel que alcanza el agua por gravedad, de manera que se necesitaba detener la circulación en el canal principal para acumular el agua en su interior y elevar el nivel para que pudiese acceder a estas tierras altas. En general, estas actuaciones están documentadas entre los siglos XV al XVIII, y con estas excepciones no parece que los sistemas se ampliasen mucho más, puesto que existían restricciones legales para ello establecidas por las propias comunas en sus ordenanzas y otros documentos (Jaubert de Passa, 1844; Glick, 1988: 345), precisamente para no tener que repartir más la dotación de agua. Además, la Huerta de Valencia estaba encajada entre su propia línea de rigidez y los marjales situados en la costa. Estos últimos, por su parte, se fueron desecando a partir del siglo XIV en adelante para ser cultivados. Pero estas nuevas tierras desecadas no tenían derecho a riego, pues solo aprovechaban el agua que sobraba a las tierras más antiguas y nunca se integraron en las comunas históricas, por lo que no se contabilizaban en los registros de las comunas para el pago de las tasas en relación con la tierra poseída, que es de donde se extraen siempre los datos de superficie irrigada por cada acequia.

Así pues, volviendo a la Tabla 4, son las ampliaciones de época moderna las que distorsionan la asignación original de filas por superficie. No obstante, como ya hemos visto, a partir de algunos datos sueltos se puede reconstruir la superficie de tierra irrigada por Tormos y Favara en época bajomedieval, y eso permite deducir, al menos para estos casos, que en la distribución original del agua del río Turia se asignó muy probablemente a cada acequia una fila de agua por cada 30 jovades de superficie irrigada. En primer lugar porque esta es la cifra que nos proporciona Tormos, que es una de las acequias que menos se había ampliado; pero, sobre todo, porque 30 jovades es la media resultante con

13. Archivo de la Comunidad de regantes de la Acequia de Favara, Capatró de la Séquia de Favara, 1741, sin catalogar. Debo agradecer a E. Guinot que me facilitase estos datos. 
las cifras del siglo XIX. Es decir, que en el Turia se hizo lo mismo que en el Mijares, pero en este último con una fila por cada 50 jovades como pudo calcular Selma (2005), y en el Turia con una fila por cada 30 jovades. Solo que ya sabemos que ambas filas son distintas, puesto que la fila de la Plana de Castelló era de tres palmos cuadrados forales $(0,154$ $\mathrm{m}^{2}$ en total) mientras que la fila de la Huerta de Valencia tenía un marco de un codo cuadrado andalusí de 30 dedos $\left(0,275 \mathrm{~m}^{2}\right)$.

Dicho esto, si hacemos los cálculos necesarios (número de filas asignadas a cada acequia según la Tabla 1, multiplicadas por 30 jovades cada una), sabremos la superficie de tierra irrigada que fue estimada para cada sistema hidráulico de la Huerta de Valencia por quien ideó este sistema de distribución (Tabla 5). Además, en la última columna encontraremos la diferencia entre la superficie irrigada teórica que debería tener cada acequia según su número de filas, y la superficie real irrigada en el siglo xix según los datos de Jaubert de Passa, lo que indica cuánto había aumentado la superficie irrigada por cada acequia.

TABLA 5

Filas de la Huerta según el reparto a 30 jovades por cada fila

\begin{tabular}{lrccc}
\hline Acequia & Filas & Superficie a 30 jov./fila & Superficies. XIX & Diferencia superficie \\
\hline Montcada & 24 & 720 & 1.066 & +346 \\
Quart & 14 & 420 & 515 & +95 \\
Tormos & 10 & 300 & 305 & +5 \\
Mislata & 10 & 300 & 283 & -17 \\
Mestalla & 14 & 420 & 387 & -33 \\
Favara & 14 & 420 & 519 & +99 \\
Rascanya & 14 & 420 & 262 & -158 \\
Rovella & 14 & 420 & 172 & -248 \\
Total & 114 & 3.420 & 3.509 & +89 \\
\hline
\end{tabular}

Fuente: Elaboración propia.

Lo primero que llama la atención es que las estimaciones de superficie irrigada realizadas anteriormente para Tormos y Favara en la Baja Edad Media cuadran casi a la perfección con la superficie teórica irrigada según su número de filas. Así, sabemos que Tormos regaba 283 jovades en 1433, y que debería regar 300 según su número de filas, lo que indica que en el siglo Xv se regaban 17 menos de la capacidad que se le había estimado inicialmente; y en el XIX, con 305 jovades según Jaubert de Passa, regaba solo 5 por encima de su capacidad teórica. Esto resulta totalmente lógico teniendo en cuenta que Tormos es, precisamente, uno de los sistemas hidráulicos más encajados que hay en la Huerta de Valencia, con Montcada rodeándolo por el norte y Rascanya-Mestalla por el 
este y el sur (Figura 1), de manera que resulta muy difícil ampliarlo excepto en aquellas zonas más altas que hubiese dentro del perímetro máximo de Tormos. Lo mismo que la acequia de Mislata, que apenas debió crecer porque está encajada entre Quart-Benàger y Favara. En el caso de esta última, la estimación realizada para época bajomedieval -eliminando ampliaciones del registro del siglo XviII- nos indica que se regaban alrededor de 429 jovades, que son solo 9 más de las 420 que podía regar con su cuota de agua según las filas asignadas. Por el contrario, en el siglo XIX, después de las ampliaciones de época moderna, regaba ya 99 jovades por encima de su máximo teórico establecido en las filas, lo que explicaría el habitual déficit de agua que tenía en época contemporánea. Como en Montcada, especialmente en su parte final del Puig y Puçol, donde las dificultades eran perennes (Glick, 1988).

\section{FIGURA 1}

Plano de la Huerta de Valencia con la superficie irrigada por cada una de sus ocho acequias en el momento de máxima expansión del espacio irrigado

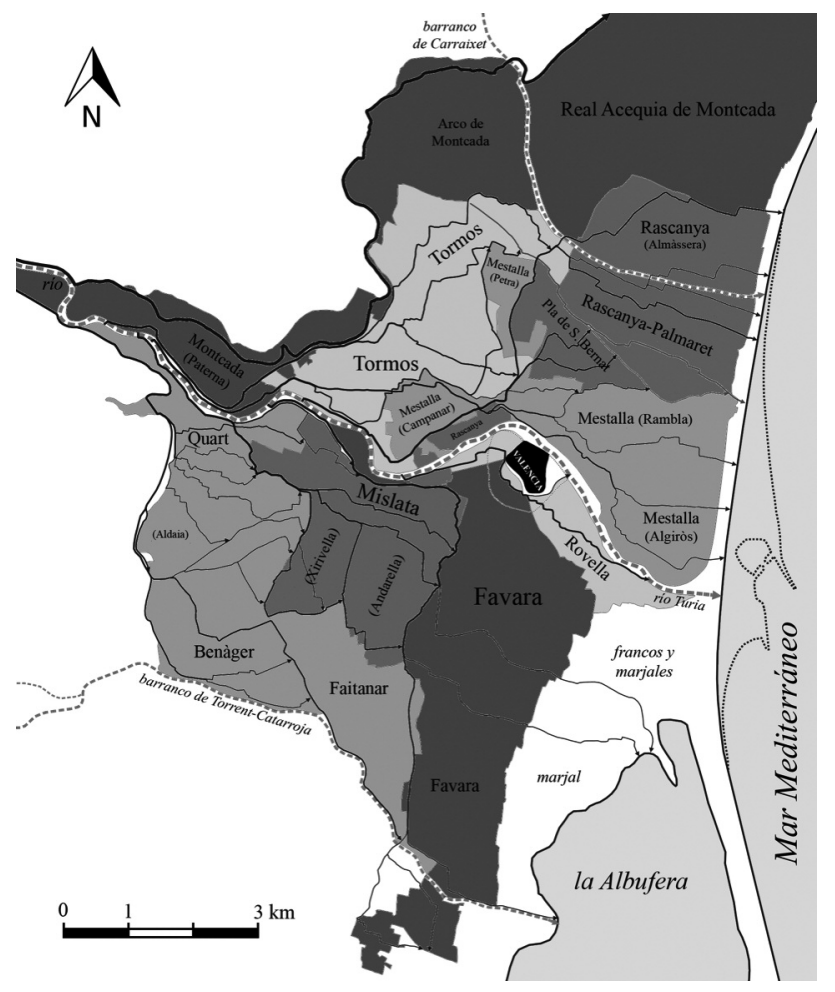

Fuente: Esquilache (2018).

La Tabla 5 nos indica, pues, que en el siglo XIX las acequias de Montcada, Quart-Benàger y Favara eran las que más habían crecido, por una simple cuestión de espacio dis- 
ponible; mientras que las de Tormos, Mislata y Mestalla todavía estaban bastante equilibradas con respecto a su supuesta cuota de agua, pues su crecimiento había sido muy limitado, estando estas dos últimas incluso por debajo de su capacidad. De hecho, esto último evidencia que la superficie original a partir del número de filas debe ser entendida como una cifra aproximada, una estimación que debieron calcular los expertos responsables de hacer la distribución del río en filas. Aunque esto ya era bastante obvio antes de conocer las cifras de superficie y hacer cálculos especulativos con ellas, puesto que dos de las acequias tenían asignadas 10 filas y hasta cinco de ellas tenían 14 filas, y es bastante lógico pensar que las superficies de todos estos sistemas no podían haber sido exactamente las mismas en ningún momento de su historia. Por otro lado, ya hemos visto que Selma (2005) advirtió que en el caso del Mijares las cifras de superficie irrigada sobre las que se había calculado el número de filas correspondientes a cada acequia en la sentencia de 1347 eran también una estimación, aunque ciertamente mucho más ajustada.

Por lo que respecta a las dos acequias cuya superficie real en el siglo XIX era muy inferior a la estimada según el número de filas, que son Rascanya y Rovella, existe una explicación bastante plausible al respecto. En el caso de Rascanya, las 262 jovades que recoge Jaubert de Passa son las censadas en la comuna, pero en realidad regaba mucha más tierra. Si observamos la Figura 1, veremos que Rascanya estaba dividida en dos zonas: al sur la del Pla de Sant Bernat -que es en realidad la antigua alquería andalusí de Rascanya, que dio nombre a toda la acequia tras la conquista cristiana, antes llamada Isbā-y al norte la zona de Almàssera y Alboraia, a ambos lados del barranco de Carraixet. Entre medio quedaban las tierras de Palmaret, con un riego combinado por la misma acequia y por una fuente natural situada junto a la localidad de Carpesa, que históricamente no han pertenecido a la comuna hasta su ingreso en el siglo xx. Pero si sumamos estas tierras no censadas a las que sí lo estaban, el resultado es una cifra muy próxima a las 420 jovades que le corresponderían con 14 filas de agua, de manera que parece muy probable que en el momento de organizar la distribución del agua del río entre las diversas acequias, esta zona fuese considerada como propia de la acequia de Rascanya y por eso le asignaron las 14 filas que tiene.

En el caso de la acequia de Rovella, que fue la acequia urbana por excelencia desde su construcción en época andalusí, hay que tener en cuenta que en sus 14 filas se incluía la parte de agua del río destinada al interior de la ciudad, conocida habitualmente como «la mola de sang i foc» (Burriel, 1971: 180). Una cantidad de agua que, como indica su nombre, se usaba para la limpieza de las alcantarillas y de la carnicería, para la extinción de incendios, así como para el riego de huertos urbanos. No sabemos cuánto era una muela de agua, pero si eran nueve filas, como parece más probable (Esquilache, 2021), 
eso quiere decir que la acequia de Rovella tenía en realidad solamente 5 filas para la irrigación de los campos de cultivo exteriores a la muralla, de manera que a 30 jovades por fila serian 150 las jovades previstas. Una cifra mucho más próxima a las 172 jovades que según Jaubert de Passa aún regaba esta acequia a principios del siglo XIX, unos años antes del inicio de la expansión urbanística de la ciudad de Valencia. Así pues, las cifras de estas dos acequias también encajan de forma razonable.

Volviendo al origen de las 138 filas, con lo que hemos visto hasta ahora no pueden datarse más allá de un genérico origen medieval, pues sabemos que existe una relación entre el número de filas de cada acequia y la superficie de tierra que riega, y sabemos que esta superficie es anterior a las ampliaciones de época moderna, pero seguimos sin saber cuándo se realizó exactamente el reparto del río. Todo apunta a un origen bajomedieval, pero teniendo en cuenta la obsesión para que todo aquello relacionado con la irrigación se mantuviese "com en temps de sarraïns", así como el hecho de que no se conozca, en principio, ningún registro documental sobre posibles cambios en la distribución de las aguas del río realizados tras la conquista, y teniendo en cuenta también que las filas de la Huerta de Valencia están basadas en un codo andalusí, no se puede descartar que las 138 filas tuviesen un origen anterior a la conquista, como suponía Glick. Pero para ver si su origen es andalusí o no, primero sería necesario saber cuánta tierra se irrigaba durante este período en la Huerta deValencia, asumiendo, evidentemente, que en época andalusí también se repartía el agua en relación con la cantidad de tierra irrigada, como indican los Fueros para la época bajomedieval.

Hasta hace poco esto era totalmente imposible, pero ahora podemos hacernos una idea aproximada de cuánta tierra se regaba en la Huerta de Valencia en época andalusí gracias al detallado análisis morfológico que se ha venido realizando en la última década sobre esta gran área irrigada (Esquilache, 2018).Y, aunque es cierto que se trata solo de una propuesta de reconstrucción basada en la morfología y, por tanto, es bastante seguro que contendrá errores, los datos obtenidos como resultado de sumar la superficie de los diversos espacios irrigados andalusíes que se han podido identificar para cada acequia (excepto Montcada, que aún no ha sido estudiada al completo) pueden servir para hacer un análisis comparativo (Figura 2). El resultado es la Tabla 6, a la que se han aplicado los mismos criterios que al resto de las tablas anteriores. 
TABLA 6

Filas de la Huerta en relación con la superficie irrigada en época andalusí

\begin{tabular}{|c|c|c|c|c|c|c|c|}
\hline Acequia & Filas & $\begin{array}{r}\% \\
\text { filas }\end{array}$ & $\begin{array}{r}\text { Superficie } \\
\text { en ha }\end{array}$ & $\begin{array}{r}\% \\
\text { superficie }\end{array}$ & $\begin{array}{r}\text { Hectáreas } \\
\text { por fila }\end{array}$ & $\begin{array}{r}\text { Superficie } \\
12 \text { ha/fila }\end{array}$ & $\begin{array}{l}\text { Diferencia } \\
\text { superficie }\end{array}$ \\
\hline Montcada & - & - & - & - & - & - & - \\
\hline Quart & 14 & $15,5 \%$ & 174,5 & $15.14 \%$ & 12,46 & 168 & $+6,5$ \\
\hline Tormos & 10 & $11,1 \%$ & 203 & $17,62 \%$ & 20,3 & 120 & +83 \\
\hline Mislata & 10 & $11,1 \%$ & 112,5 & $9,76 \%$ & 11,25 & 120 & $-7,5$ \\
\hline Mestalla & 14 & $15,5 \%$ & 180 & $15,62 \%$ & 12,86 & 168 & +12 \\
\hline Favara & 14 & $15,5 \%$ & 366,5 & $31,8 \%$ & 26,18 & 168 & $+198,5$ \\
\hline Rascanya & 14 & $15,5 \%$ & 106,5 & $9,24 \%$ & 7,60 & 168 & $-61,5$ \\
\hline Rovella & 14 & $15,5 \%$ & 9 & $0,78 \%$ & 0,64 & 168 & -159 \\
\hline Total & 90 & $100 \%$ & 1.152 & $100 \%$ & 12,8 & 1.080 & +72 \\
\hline
\end{tabular}

Fuente: Elaboración propia con datos tomados de Esquilache (2018).

\section{FIGURA 2}

Plano de la Huerta de Valencia en época andalusí, justo antes de la conquista cristiana, con pequeños espacios irrigados y grandes intersticios no irrigados

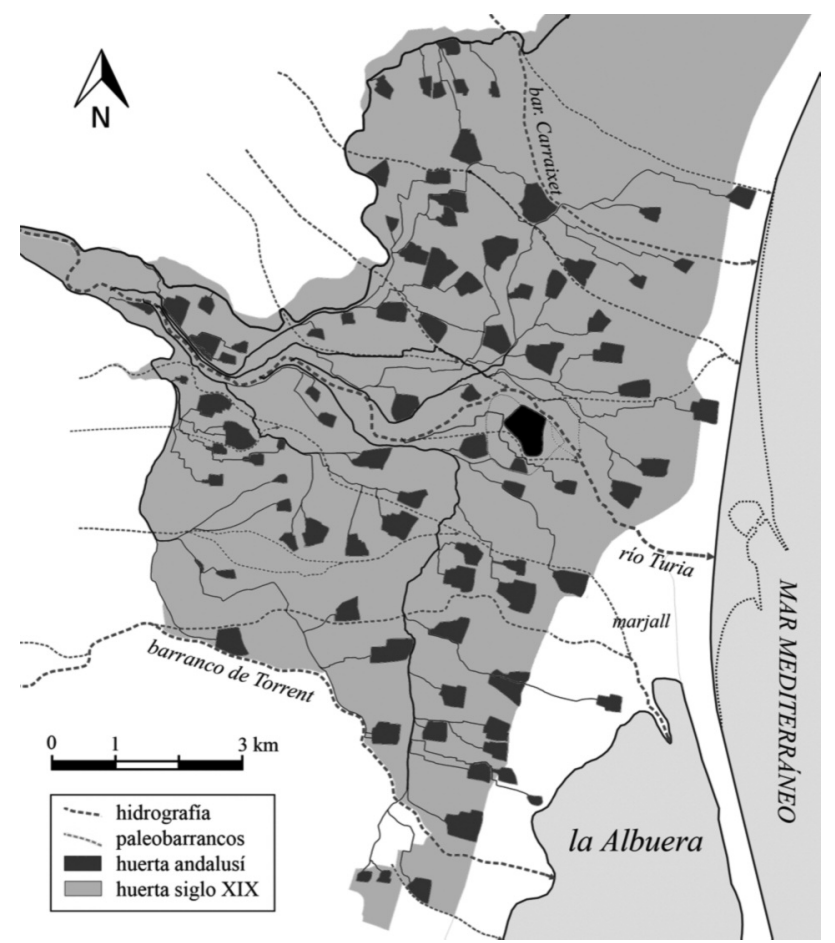

Fuente: Esquilache (2018). 
Llama mucho la atención la enorme coincidencia entre el porcentaje de filas de agua y el porcentaje de tierra irrigada en el caso de Quart y Mestalla, y la cercanía de Mislata. Pero la coincidencia se desvanece totalmente en el caso de Tormos y Favara, que son precisamente las dos acequias con una mayor superficie irrigada. De hecho, en el caso de Favara especialmente, la desproporción es desorbitada, pues incluso quitando los espacios irrigados que seguramente son más tardíos y los que están dentro del marjal (Figura 2), la cifra de superficie real en época andalusí ni siquiera se aproximaría a la teórica que se le puede suponer a Favara a razón de 12 hectáreas de tierra irrigada por cada fila de agua, que sería lo más lógico de acuerdo con los datos de Quart y Mestalla, y que es lo que podemos ver en la penúltima columna. También hay una gran diferencia entre la tierra real y la teórica en el caso de Rascanya y Rovella, aunque en el sentido contrario, puesto que la superficie real irrigada por estas dos acequias en época andalusí era muy pequeña, por no decir ínfima en el caso de Rovella porque fuera de las murallas de la ciudad esta solo regaba en la zona de Russafa. Por otro lado, una fila por cada doce hectáreas (unas 4 jovades forales), tal como sugieren los datos de las acequias de Quart, Mestalla y Mislata, es una cantidad ingente de agua que no tiene sentido para tan poca superficie de tierra irrigada, de manera que la coincidencia de porcentajes en el caso de estas tres acequias mencionadas parece más bien una casualidad que otra cosa, mientras que Tormos, Favara, Rascanya y Rovella confirman de forma bastante plausible que las tradicionales 138 filas de agua del río Turia en la Huerta de Valencia no tienen un origen andalusí, a pesar de estar basadas en un codo establecido en este período.

Así pues, si el sistema de distribución del agua en la Huerta de Valencia, con sus 138 filas, es anterior a época moderna, cuando casi todos los sistemas hidráulicos fueron ligeramente ampliados, y no es de origen andalusí como acabamos de ver, no queda más remedio que considerar que es bajomedieval. Además, de un momento bastante temprano tras la conquista, como sugiere el hecho de que se usase aún una fila con el marco de un codo andalusí, y no una fila basada en las nuevas medidas de longitud establecidas por Jaime I en los Fueros de Valencia de 1238. De hecho, ya hemos visto que más adelante la fila pasó a ser el agua que pasa por un orificio con el marco de un palmo cuadrado foral valenciano, y que la fila de la Plana de Castelló que se aplica en la sentencia del Mijares de 1347 es de tres palmos cuadrados forales, aunque no podemos saber cuándo ni por qué se aplicaron estos cambios que no aparecen en ningún documento conocido.

En definitiva, todo apunta a que las 138 filas de la distribución tradicional del agua del río Turia entre las ocho acequias de la Huerta de Valencia es consecuencia del proceso de repartimiento de la tierra producido inmediatamente después de la conquista, bien conocido a partir del llamado Llibre del Repartiment y otros documentos coetáneos (Guinot, 2007). De hecho, aunque tradicionalmente se ha aducido una gran continuidad en 
la irrigación antes y después de la conquista cristiana, ahora sabemos que el proceso de reparto de la tierra conllevó profundos cambios físicos en los sistemas hidráulicos de la Huerta de Valencia al llevarse a cabo grandes ampliaciones de la superficie irrigada, producidas como consecuencia de la puesta en cultivo de los intersticios existentes entre los espacios irrigados andalusíes (Figura 2), lo que condujo a la configuración de la Huerta tal como la conocemos en la actualidad (Guinot \& Esquilache, 2012; Esquilache, 2018).

Por tanto, si los cambios físicos en la Huerta de Valencia respecto de la cantidad de tierra irrigada fueron tan grandes, es evidente que la cantidad de agua destinada a cada sistema de irrigación también tuvo que cambiar. Si no se hubiese hecho así, no solo habría habido grandes diferencias de abastecimiento de agua entre unos sistemas y otros tras las ampliaciones realizadas, con las consecuentes protestas de sus usuarios -puesto que antes de la conquista la superficie irrigada era muy distinta en cada sistema-, sino que además hubiese sido ilegal, de acuerdo con el fuero de Jaime I que hemos visto antes cuando se hablaba de la distribución del río Mijares. Con todo, ello no es óbice para admitir, aunque no haya pruebas y no pase de ser una hipótesis, que el nuevo sistema de distribución en 138 filas fue, probablemente, la modificación de un sistema previo muy similar, que ya debía existir en época andalusí, puesto que la fila como medida de aforo tiene este origen. Pero una cosa es el sistema de distribución, y otra la cantidad de agua que corresponde a cada acequia.

\section{CONCLUSIONES}

De todo lo dicho hasta ahora se pueden extraer al menos dos conclusiones básicas. En primer lugar, que la hipótesis de reconstrucción del sistema de distribución del agua del río Turia para época andalusí planteada por Glick no parece que pueda ser correcta, puesto que, entre otras razones, obvia la naturaleza principal de la fila como una medida de agua concreta, y no proporcional o temporal como se venia defendiendo hasta ahora. $\mathrm{Y}$, en segundo lugar, se puede concluir que el sistema de distribución tradicional del agua de la Huerta de Valencia, con sus 138 filas, aunque posiblemente estuvo basado en un sistema similar más antiguo, de época andalusí, probablemente fue una reelaboración posterior a la conquista cristiana del siglo XIII, de acuerdo con los datos de superficie irrigada disponibles.

Esto es totalmente lógico teniendo en cuenta los profundos cambios físicos que se produjeron en la Huerta de Valencia a partir de la rendición de la medina andalusí ante las huestes feudales de Jaime I. Especialmente por lo que se refiere al gran aumento de la superficie irrigada como consecuencia del reparto de tierras entre los conquistadores -apro- 
ximadamente entre los años 1238 y 1242 (Guinot, 2007)-, y a la inmediata puesta en cultivo de todo el espacio disponible dentro del perímetro de las acequias, que antes de la conquista no estaba todo irrigado (Figura 2). De hecho, de haberse mantenido el anterior sistema andalusí de distribución del agua, los sistemas de irrigación existentes en la Huerta hubiesen quedado totalmente descompensados ante un aumento diferente de la superficie irrigada por cada uno de ellos.Y esto no solo hubiese implicado protestas, sino que, además, hubiese ido en contra de la propia ley foral que exigía una distribución proporcional del agua en relación con la superficie irrigada.

Sin embargo, más allá de la identificación cronológica del origen de este sistema de distribución tradicional del agua de riego del río Turia -más o menos segura a partir de las evidencias disponibles y los cálculos realizados, a pesar de no disponer de pruebas documentales-, son mucho más importantes las implicaciones sociales que conlleva este cambio. Las investigaciones que se están llevando a cabo últimamente apuntan cada vez con más seguridad que la continuidad en la gestión de la irrigación antes y después de la conquista es más aparente que real, y que la conocida frase "com en temps de sarraïns" tiene unas implicaciones muy diferentes a las que tradicionalmente se habían supuesto (Guinot, 2008; Torró, 2013; Retamero, 2014; Esquilache, 2019).

En este sentido, los cambios de uso y de gestión de la Huerta introducidos por los conquistadores, que en realidad son consecuencia de los grandes cambios físicos realizados, implican entre unos y otros que la transformación de los espacios irrigados capturados a la sociedad andalusí fue tan profunda que se podrían considerar como una reconstrucción casi completa. A pesar de que todo aquello que ya existía antes de la conquista continuó existiendo, y por eso hoy en día aún puede ser identificado mediante análisis morfológicos (Esquilache, 2018). Pero es ya evidente que los espacios irrigados de los andalusíes y de los cristianos no eran iguales, ni se usaban y gestionaban del mismo modo, puesto que sus sociedades tampoco funcionaban de la misma manera y la gestión de la irrigación se adaptaba a cada una de ellas.

\section{AGRADECIMIENTOS}

Debo agradecer encarecidamente los comentarios de los evaluadores de la revista Historia Agraria, que, sin duda, han contribuido a mejorar este texto. Este trabajo forma parte del proyecto "La gestión de la herencia agraria andalusí: Modificaciones y transformaciones realizadas en los espacios irrigados del Reino de Valencia después de la conquista cristiana, siglos XIII-XVI (UJI-A2018-07)", financiado por la Universitat Jaume I. 


\section{REFERENCIAS}

Aymard, M. (1864). Irrigations du Midi de l'Espagne. Paris: E. Lacroix.

Borrull, F. X. (1831). Tratado de distribución de la aguas del río Turia y del Tribunal de los Acequieros de la Huerta de Valencia. Valencia: Impr. B. Monfort.

Burriel, E. (1971). La Huerta de Valencia, zona sur: Estudio de Geografía Agraria. Valencia: Institución Alfonso el Magnánimo.

CAVANILLES, A. J. (1795). Observaciones sobre la historia natural, geografía, agricultura poblaciones y frutos del Reyno de Valencia. Madrid: Impr. Real.

EL FAïz, M. (2005). Les maîtres de l'eau: Historie de l'hydraulique arabe. Arles: Actes Sud. ESQUILACHE, F. (2014). La cuestión de la proporcionalidad en la distribución del agua de riego: El caso de la acequia de Favara (Huerta de Valencia). En C. SANCHIS et al. (Coords.), Irrigation, Society, Landscape:Tribute to Thomas Glick (pp. 50-69).Valencia: Universidad Politécnica de Valencia.

Esquilache, F. (2018). Els constructors de l'Horta de València: Origen, evolució i estructura social d'una gran horta andalusina. Valencia: Universitat de València.

ESQUILACHE, F. (2019). Una herència reconstruïda: Canvis físics i institucionals en les hortes fluvials andalusines després de la conquesta cristiana. En E.VICEDO (Ed.), IX Congrés sobre sistemes agraris, organització social i poder local: Recs històrics: Pagesia, història i patrimoni (pp. 449-474). Lleida: Institut d'Estudis Ilerdencs.

ESQUILACHE, F. (2021). La 'fila' de agua valenciana y otras medidas de aforo: La verdadera naturaleza de un sistema de medición de caudales de origen andalusí. Espacio, tiempo y forma. Serie III, Historia medieval, (34), 293-322.

García Edo, V. (1994). Derechos históricos de los pueblos de la Plana a las aguas del río Mijares: Colección documental de los siglos XIII a XX. Castellón de la Plana: Diputació de Castelló.

GLIck, T. F. (1988). Regadio y sociedad en laValencia medieval.Valencia: Del Cenia al Segura.

GLICK, T. F. (1989). Las técnicas hidráulicas antes y después de la conquista. En Consell Valencià de Cultura (Ed.), En torno al 750 aniversario: Antecedentes y consecuencias de la conquista de Valencia (pp. 53-71). Tomo I. Valencia: Generalitat Valenciana.

GLICK, T. F. (2007). Paisajes de conquista: Cambio cultural y geográfico en la España medieval. Valencia: Universitat de València.

GuINot, E. (2005). L'Horta de València a la Baixa Edat Mitjana: De sistema hidràulic andalusí a feudal. Afers, (51), 271-300.

GuINot, E. (2007). El repartiment feudal de l'Horta de València al segle XIII: Jerarquització social i reordenament del paisatge rural. En E. GuINOT \& J. TORRó (Eds.), Repartiments a la Corona d'Aragó (pp. 115-199). Valencia: Universitat de València. 
Guinot, E. (2008). "Com en temps de sarraïns»: La herencia andalusí en la huerta medieval de Valencia. En I. Del Val \& O. VillanueVa (Eds.), Musulmanes y cristianos frente al agua en la ciudades medievales (pp. 173-193). Santander: Universidad de Castilla-La Mancha/Universidad de Cantabria.

GuINOT, E. \& EsQUILACHE, F. (2012). La reorganización del paisaje agrario en la Huerta de Valencia después de la conquista cristiana: El sistema hidráulico y el parcelario de Montcada y Benifaraig en el siglo xiII. Debates de Arqueología Medieval, (2), 229-276.

Guinot, E. \& Selma, S. (2005). Les séquies de l'Horta Nord de València: Mestalla, Rascanya i Tormos. Valencia: Generalitat Valenciana.

Jaubert de PASSA, F. (1844). Canales de riego de Cataluña y reino deValencia. 2 vols. Valencia: Impr. B. Monfort.

LÓPEZ GómEZ, A. (1975). El origen de los riegos valencianos II: La división del agua. Cuadernos de Geografía, (17), 1-38.

Manzano, E. (1986). El regadío en al-Andalus: Problemas en torno a su estudio. En la España medieval, (8), 617-632.

RETAMERo, F. (2014). ¿'Como solía en tiempos de moros'?: Los riegos después de las conquistas: El caso de Casarabonela, Málaga (siglos XV-XVI). En C. SANCHIS et al. (Coords.), Irrigation, Society, Landscape. Tribute to Thomas F. Glick (pp. 116-131).Valencia: Universidad Politécnica de Valencia.

SELma, S. (2005). Aigua i terra a la Plana del Millars: La sentència arbitral de 1347. Afers, 20 (51), 397-406.

Thoumin, R. (1934). Notes sur l'aménagement et la distribution des eaux à Damas et dans sa Ghouta. Bulletin d'Études Orientales, (4), 1-26.

ToRRó, J. (2013). Canteros y niveladores: El problema de la transmisión de las técnicas hidráulicas andalusíes a la sociedades conquistadoras. Miscelánea Medieval Murciana, (37), 209-231.

Tresse, R. (1929). L'irrigation dans la Ghouta de Damas. Revue des Études Islamiques, (3), 459-573. 